\title{
A CONCEPTUAL DESIGN FOR A SPACEBORNE 3D IMAGING LIDAR
}

\author{
John J. Degnan \\ Code 920.3, Geoscience Technology Office \\ NASA Goddard Space Flight Center \\ Greenbelt, MD 20771 USA \\ jjd@ltpmail.gsfc.nasa.gov
}

\begin{abstract}
First generation spaceborne altimetric approaches are not well-suited to generating the few meter level horizontal resolution and decimeter accuracy vertical (range) resolution on the global scale desired by many in the Earth and planetary science communities. The present paper discusses the major technological impediments to achieving few meter transverse resolutions globally using conventional approaches and offers a feasible conceptual design which utilizes modest power $\mathrm{kHz}$ rate lasers, array detectors, photoncounting multi-channel timing receivers, and dual wedge optical scanners with transmitter point-ahead correction.
\end{abstract}

\section{INTRODUCTION}

Over the past two decades, there has been an increasing interest in, and proliferation of, airborne laser altimeters, which measure the roundtrip time of flight of a laser pulse from an aircraft to the surface. High speed optical scanning devices of various types have been used to direct a train of high repetition rate $(-10 \mathrm{kHz})$ laser pulses to an array of points on the ground. Combined with GPS Differential positioning, these instruments routinely provide 3D topographic maps over wide areas with point spacings on the order of a few meters and vertical (range) accuracies on the order of a decimeter. The applications for these devices are numerous and include commercial surveying of populated areas, biomass estimation and forestry management through the measurement of tree canopies and subcanopies, changes in sea and lake levels, the determination of hydrological runoff routes with application to flood control and hazard estimation, and even personal security applications. Sophisticated software packages, tailored to specific applications, can, for example, strip the surface of manmade structures and vegetation, revealing hidden fault lines of interest to geologists and geophysicists. Airborne instruments can provide useful local, and even regional, data, but many in the Earth science community desire comparable data on a global scale.

A small number of laser altimeters have operated in space. Altimeter data from three manned Apollo missions and the 1994 Clementine mission has recently been processed to provide a crude topographical map of the Moon [Smith et al, 1997]. In the early 1970's, three of NASA's manned Apollo missions $(15,16$, and 17) carried a low repetition rate ( 16 to 32 second fire interval) ruby laser altimeter to the Moon and provided approximately 7080 measurements of the command module height relative to the lunar topography. In 1994, the Clementine Mission operated for about two months at a 
substantially higher, but still low, laser fire rate of $2 \mathrm{~Hz}$ and provided a more robust set of 70,000 altimetric measurements during approximately two months in lunar orbit. A similar altimeter on the Near Earth Asteroid Rendezvous (NEAR) mission has recently provided a detailed topographic map of the asteroid EROS.

The largest body mapped to date by a laser is the planet Mars. NASA's Mars Orbiting Laser Altimeter (MOLA) instrument [Ramos-Izquierdo et al, 1994] on the Mars Global Surveyor (MGS) spacecraft recently completed the first global topographic map of a planet from a near-polar orbit using an actively Q-switched, diode-pumped Nd:YAG laser operating at a repetition rate of $10 \mathrm{~Hz}$. With an orbital ground velocity on the order of $3 \mathrm{~km} / \mathrm{sec}$, the along-track spacing between consecutive measurements was about 300 $\mathrm{m}$. Like other missions, the MOLA instrument did not include a cross-track scanner, and hence only terrain directly below the spacecraft was interrogated under normal operating conditions. Periodically, however, the entire spacecraft was tilted via ground command to obtain data at or near the Martian poles. As with any near-polar orbit, the spatial concentration of range measurements is large at higher planetary latitudes and falls off dramatically as one approaches the Martian equator. Nevertheless, MOLA provided the most accurate global vertical height datum ever achieved from planetary orbit and was an unqualified engineering and scientific success.

Upcoming NASA missions, such as the Geoscience Laser Altimeter System (GLAS) [Abshire et al, 2000] and the Messenger Laser Altimeter (MLA), will attempt a similar feat from orbits about Earth and Mercury respectively. Like MOLA, GLAS will produce 2D profiles with consecutive along-track data points separated by about 180 meters, resulting from a laser fire rate of $40 \mathrm{~Hz}$ and an orbital ground speed on the order of 7 $\mathrm{km} / \mathrm{sec}$.

As we will demonstrate in the next section, first generation altimetric approaches are not well-suited to generating the few meter level horizontal resolution and decimeter precision vertical (range) resolution on the global scale desired by many in the Earth and planetary science communities. The present paper will attempt to address the major technological impediments to achieving few meter transverse resolutions globally using conventional approaches and offer a feasible conceptual design which utilizes modest power $\mathrm{kHz}$ rate lasers, array detectors, photon-counting multi-channel timing receivers, and dual wedge optical scanners.

\section{CHALLENGES TO GLOBAL TOPOGRAPHY VIA CONVENTIONAL ALTIMETRY}

As described previously, first generation spaceborne altimeters have been characterized by a laser operating in the infrared $(1064 \mathrm{~nm})$ at a few tens of $\mathrm{Hz}$ with moderate output energies ( 50 to $100 \mathrm{~mJ}$ ), a telescope in the 50 to $100 \mathrm{~cm}$ range, and a single element (i.e. non-pixellated) detector which detects and processes multi-photon returns from the surface. On bare terrain, the signal waveforms reflect the slope and surface roughness within the laser footprint (typically several tens of meters in diameter) as well as any false slopes due to pointing error. On Earth, the presence of manmade buildings and 
volumetric scatterers (such as tree canopies or other vegetation) generally makes waveform interpretation more complex and difficult.

Clearly, one major challenge to the conventional approach is the sheer number of measurements required over a nominal mission lifetime of two to three years. For example, in order to generate a $5 \mathrm{~m} \times 5 \mathrm{~m}$ vertical grid map of Mars, which has a mean volumetric radius of $3390 \mathrm{~km}$, over 7 trillion individual range measurements are required, even if one makes the unrealistic assumption that no ground spatial element is measured twice. In any realistic mission, the actual number of range measurements will be significantly larger since an instrument designed to provide contiguous coverage at the planetary equator will oversample the higher latitudes where the ground tracks are more narrowly spaced. Over the multiyear life of the mission, MOLA produced less than one billion range measurements to the surface. The laser energy fell from an initial value of $50 \mathrm{~mJ}$ to about $20 \mathrm{~mJ}$ as pump diode bars failed before an unrelated failure in an onboard oscillator, which triggered the laser, silenced the transmitter forever. If one were to simply scale conventional approaches, one would clearly face severe prime power, weight, and instrument longevity issues.

A second technical challenge is the high ground speed of the spacecraft (about $3 \mathrm{~km} / \mathrm{sec}$ for a nominal $300 \mathrm{~km}$ altitude Mars orbit) coupled with the need to incorporate a scanner to cover the large area between adjacent ground tracks, especially near the equator. At a nominal altitude of $300 \mathrm{~km}$, the satellite would have an orbital period about Mars of approximately 113 minutes. Thus, a three year mission would produce 13,910 orbits or 27,820 equator crossings with an average spacing between ground tracks at the equator of 766 meters. The latter spacing corresponds to about 154 resolution elements $(\Delta=5 \mathrm{~m})$ in the cross-track direction between adjacent ground tracks and further implies a minimum cross-track scan angle of about 0.15 degrees. For truly contiguous coverage using a conventional single element detector, these 154 cross-track measurements must be completed in the time it takes the spacecraft to move one resolution element in the alongtrack direction, or within $1.67 \mathrm{msec}$. This implies a rather daunting laser fire rate of 92.4 $\mathrm{kHz}$. Furthermore, a uniformly rotating mechanical scanner, for example, must complete a half cycle of its movement within the same $1.67 \mathrm{msec}$ period, i.e. an impossible $300 \mathrm{~Hz}$ (18,000 RPM) rate. While alternative non-mechanical scanners, such as electro-optic or acousto-optic devices, are capable of very high scanning speeds and have no moving parts, they fall far short of the angular range requirements, are highly limited in their useful aperture, and require fast high voltage or high RF power drivers.

An additional technical challenge stems from the high laser fire rate and the long pulse time of flight (TOF). At $300 \mathrm{~km}$ altitude, the laser pulse completes a roundtrip transit to the surface in $2 \mathrm{msec}$. Thus, for laser fire rates in excess of $500 \mathrm{~Hz}$, multiple pulses will be in flight simultaneously. In principle, it is easy to associate the correct return pulse with the appropriate outgoing pulse provided the roundtrip satellite-to-surface TOF is known a priori to well within a single laser fire interval. For the $92.4 \mathrm{kHz}$ rate derived previously, however, approximately 185 pulses would be simultaneously in transit, and it would be necessary to have a priori orbital knowledge at the $1.6 \mathrm{~km}$ level in order to unambiguously tie a given surface return to the appropriate output pulse. While such a 
navigation accuracy might be easy to achieve in Earth orbit using either Global Positioning System (GPS) receivers or Satellite Laser Ranging (SLR) to passive reflectors on the spacecraft [Degnan, 1993], it would likely be a much more difficult challenge in orbits about extraterrestrial bodies.

A second technical problem associated with the longer pulse TOF from orbit is related to "transmitter point-ahead", i.e. the offset between the center of the laser beam at the surface and where the receiver is looking one $2 \mathrm{msec}$ round trip transit time later. For an unscanned system, the offset due to a $3 \mathrm{~km} / \mathrm{sec}$ spacecraft ground velocity is only $6 \mathrm{~m}$ (slightly more than one resolution element) in the along-track direction and can be easily accommodated, either by a fixed offset of the transmitter in the positive along-track direction or by a modest increase in the receiver field of view (FOV). In our current example, however, the scanner must complete over half a cycle of its scan within the pulse TOF. Thus, the receiver FOV must be opened up to span the full 0.15 degree separation $(766 \mathrm{~m})$ between ground tracks in the cross-track dimension while the laser illuminates only a $5 \mathrm{~m}$ diameter circle within that FOV and defines the ground resolution element being interrogated. This approach greatly increases the solar background noise incident on the detector during local daytime operations relative to the unscanned case and elevates the laser output energy requirements for good discrimination of the signal. An alternative low noise approach would be to independently steer the transmitter and receiver, and we discuss efficient means of doing this in later sections.

A final concern is the high data rate associated with contiguous mapping. At a nominal altitude of $300 \mathrm{~km}$ and a vertical range resolution of $10 \mathrm{~cm}$, one would need to transfer 22 bits per range measurement. At our $92.4 \mathrm{kHz}$ laser fire rate, this generates an average data rate of over $2 \mathrm{Mbits} / \mathrm{sec}$, which needs to be stored onboard and transmitted to an Earth station at regular intervals as spacecraft to Earth visibility permits. While this data rate does not represent a major challenge for Earth, or even lunar missions, it does tax the capabilities of current microwave systems over interplanetary distances, and one must therefore either consider some form of data compression or move to higher bandwidth communications systems, perhaps in the optical regime.

\section{THE NEED FOR ARRAY DETECTORS}

The unrealistically high laser fire rates and scan rates described in the previous section can only be ameliorated by making many spatially resolved measurements within a single pulse. This can be accomplished by replacing the single element detector with a highly pixellated array detector. The ground scene is then imaged by the receiver telescope onto the array such that each pixel records the laser photon returns from a single $5 \mathrm{~m} \times 5 \mathrm{~m}$ resolution element on the planetary surface (along with noise) and inputs them into a separate timing channel. Of course, one must now provide a multi-channel timing receiver with the number of channels equal to the number of pixels, but this can be accomplished within a reasonable volume and weight $(<2.5 \mathrm{~kg})$ for a receiver having on the order of 100 timing channels or less [Beaghler, 2001]. 
Examples of high speed detectors suitable for precise multichannel ranging include segmented anode photomultipliers (PMT's) and Avalanche Photodiode Arrays (APD's). The former are relatively inexpensive, can be obtained commercially with up to 100 anode elements $(10 \times 10)$, and have single photon sensitivity, large active apertures, subnanosecond risetimes, and high internal gains $\left(5 \times 10^{6}\right)$. Additionally, microchannel plate PMT's offer low transit time jitter, due to the highly restricted electron multiplication path, and zero dead time, since the likelihood of multiple photons hitting the same microchannel is exceedingly small. When equipped with conventional bi-alkali or multi-alkali cathodes, PMT's typically have quantum efficiencies on the order $10 \%$ to $15 \%$ at $532 \mathrm{~nm}$, a wavelength corresponding to the second harmonic of the Nd:YAG laser. More exotic cathodes such as GaAs and GaAsP permit visible QE's in the $30 \%$ and $40 \%$ range respectively whereas photon counting detectors at the fundamental Nd:YAG wavelength of $1064 \mathrm{~nm}$ are typically an order of magnitude less efficient when used in photon counting mode. High speed APD arrays operating in photon-counting or Geiger mode are typically more expensive and less mature technically than PMT's, have relatively long dead times ( $\sim 50 \mathrm{nsec}$ ) even with active quenching circuits, and the dead space created in the active aperture by the need for these circuits must often be compensated for by the use of microlens arrays. On the other hand, APD arrays offer the promise of still higher quantum efficiencies in the visible, on the order of $50 \%$ to $70 \%$, as well as larger array sizes ( $32 \times 32$ or greater). Various high efficiency, hybrid devices such as IPD's are also available from Intevac Corporation.

For truly contiguous and photon-efficient mapping, the laser beam properties must be chosen to provide maximum overlap with the projected ground image of the detector array such that there is a high probability of recording one or more signal photons per pixel per laser fire. Furthermore, solar background noise from areas not illuminated by the laser must be kept to a minimum. Although it is possible to consider both onedimensional (linear) and two-dimensional arrays, we will demonstrate in this section that the second option is the easiest to implement.

The linear array, or "pushbroom", approach has the advantages that it would eliminate the need for an optical scanner and greatly simplify the accommodation of transmitter point ahead. However, we would require 154 linear pixels (and corresponding timing channels) to cover our nominal $766 \mathrm{~m}$ spacing between adjacent ground tracks at Mars with $5 \mathrm{~m}$ resolution. Furthermore, efficient illumination demands the use of cylindrical and/or fiber optics to convert the nominally circular laser beam to a more efficient linear strip of light on the ground ( $766 \mathrm{~m} \times 5 \mathrm{~m}$ in our Mars example). For effective noise control, the receiver must also be configured to image only the light emanating from the same strip onto the array in order to eliminate any additional background noise from non-illuminated areas.

An alternative pushbroom approach, which eliminates the need for exotic optics, would be to use $\mathrm{m}$ laser transmitters with a rectangular $\mathrm{M} \times \mathrm{N}$ array viewing the area illuminated by all of the independent transmitters. Clearly, a square array provides a more efficient overlap with a nominally circular laser beam than a rectangular array. Each transmitter would therefore be pointed to fill the field of view of a $\mathrm{N} \times \mathrm{N}$ sub-array within the larger $\mathrm{M} \times \mathrm{N}$ rectangular array. Each laser would then fire at a repetition rate equal to the 
ground velocity divided by the linear dimension of the grid projected onto the ground, or $v_{g} / N \Delta=600 \mathrm{~Hz} / \mathrm{N}$. However, the condition $\mathrm{M}=\mathrm{m} \times \mathrm{N} \sim 154$ would lead to a very large number of independent ranging components including $\mathrm{m}$ transmitters and $\mathrm{mN}^{2}$ detectors, amplifiers, discriminators, and timing channels. For $\mathrm{N}=10$, one would need 16 transmitters operating at $60 \mathrm{~Hz}$ and 1600 detector pixels and timing channels.

The simplest approach, at least conceptually, is to use a single square two-dimensional array and an optical scanner as in Figure 1. If one assumes an NxN detector array, a single laser pulse can in principle measure the range to $\mathrm{N}^{2}$ resolution elements, thereby reducing the laser repetition rate and the number of laser fires required to obtain contiguous global coverage by the same factor. It also relaxes the angular beam divergence requirement on the laser by a factor of $N$, which in turn reduces the aperture areas of the transmitter telescope and scanner by $\mathrm{N}^{2}$ with corresponding benefits in overall instrument weight, volume, and prime power usage. Furthermore, for a given spacecraft ground speed, it now takes $\mathrm{N}$ times as long for the spacecraft to traverse the receiver FOV in the along track direction, and the required scanner frequency for a contiguous map is therefore reduced by a factor of $\mathrm{N}$.

Assuming a value $\mathrm{N}=10$ in our Mars example, a single pulse will now interrogate a 50 $\mathrm{m} \times 50 \mathrm{~m}$ area on the ground or 100 resolution elements. The laser fire rate can therefore be reduced by two orders of magnitude from $92.4 \mathrm{kHz}$ to less than a $\mathrm{kHz}(924 \mathrm{~Hz})$, and the scanner frequency can be reduced from $300 \mathrm{~Hz}(18,000 \mathrm{RPM})$ to a more manageable $30 \mathrm{~Hz}$ (1800 RPM). Furthermore, the required aperture for the transmitting optics and scanner is reduced by two orders of magnitude in area, relative to the single element detector case, due to the relaxed beam divergence enabled by the array detector. At 300 $\mathrm{km}$ altitude, a $50 \mathrm{~m}$ diameter laser ground spot can be produced from a final transmit aperture approximately $1 \mathrm{~cm}$ in diameter assuming a laser operating in its lowest order TEM $_{00}$ spatial mode.

Although the reduced scan rate also decreases the "point-ahead" displacement between the transmitter and receiver by a factor of 10 , the displacement can still be large (up to $\sim 100 \mathrm{~m}$ ) relative to the new and enlarged $50 \mathrm{~m}$ laser spot diameter. Since enlarging the transmitter spot on the ground to ensure a partial overlap with the array would be grossly inefficient, the transmitter and receiver FOV's must be independently steered so they tightly overlap at all times during the scan. A simple scanner design with "point-ahead" compensation is described in the next section.

\section{DUAL WEDGE SCANNER WITH TRANSMITTER POINT-AHEAD}

A variety of optical scanners have been used in airborne lidars and altimeters. To the author's knowledge, all have been mechanical in nature due to the need for wide angular sweeps for maximum areal coverage at typical aircraft altitudes. Spaceborne lidars place additional constraints on the type of scanner used. Clearly, weight, size, reliability, flexibility, suitability of the scan pattern, and prime power requirements are all important factors in choosing a scanner for space use. 
In space applications, uniformly rotating devices are generally viewed by aerospace engineers as more reliable and efficient than other mechanical devices. First of all, they are the simplest to implement, monitor, and control. Secondly, rotating scanners tend to be power efficient since, once rotating, the force applied is devoted to simply overcoming bearing or gear/belt friction. Alternative scanner types, such as a programmable two axis mirror, may offer greater flexibility in providing a more optimum scan pattern, but they must be made larger to avoid aperture vignetting and must also undergo more complex motions characterized by constant accelerations and decelerations. As a result, they consume more prime power while introducing more mechanical stress, volume, and mass.

Rotational scanners include reflective devices, such as spinning mirrors or polygons, as well as transmissive devices, such as spinning optical wedges or holographic elements. Unfortunately, conservation of total angular momentum dictates that any net angular momentum introduced by the rotating scanner will be balanced by an equal induced angular momentum in the spacecraft, which is clearly undesirable from a spacecraft control standpoint. Thus, optical scanners that impart no net angular momentum are the preferred approach for spacecraft missions, and this can be achieved through the use of counter-rotating optical elements having equal but opposite angular momenta.

Figure 2 describes a transmissive optical scanner composed of two equivalent optical wedges which are rotated at the same angular speed, but in opposite directions, by a common rotor. The angular range of the scan and the ground pattern can be easily optimized for a given application by adjusting the magnitude of the cone half-angle, $\alpha$, produced by an individual optical wedge and the relative starting phase of the plates, $\Delta \varphi$. The instantaneous position of the receiver FOV on the ground at time $t$ is then given by the equations:

$$
\begin{aligned}
& x(t)=v_{g} t+R \tan \alpha[\cos \omega t+\cos (-\omega t+\Delta \varphi)] \\
& y(t)=R \tan \alpha[\sin \omega t+\sin (-\omega t+\Delta \varphi)]
\end{aligned}
$$

where $v_{g}$ is the spacecraft ground velocity in the positive $\mathrm{x}$-direction and $\omega$ is the angular velocity of the counter-rotating plates. As described in Figure 2, a single motor can be used to drive both plates via a simple gearing arrangement, which also maintains the relative phase between the two plates indefinitely. Choosing $\Delta \varphi=90^{\circ}$ provides a fairly uniform scan pattern for mapping as illustrated in Figure 3.

By the time the receiver views the photons returning from the surface, each of the optical wedges has advanced in phase, relative to the time of laser fire, by an approximate angle $\Delta \phi=2 R \omega / c$ where $\omega$ is the angular frequency of the rotating plate and $2 R / c$ is the nominal roundtrip transit time of the pulse to the surface. Thus, the receiver FOV is displaced from the area of illumination on the ground, and, at spacecraft altitudes, this displacement can be quite large for fast-moving scanners. In order to compensate for transmitter point-ahead, each wedge consists of a central and an annular portion. The inner central wedge scans the transmitter while the outer annular wedge scans the 
receiver. Transmitter point-ahead is accomplished by advancing the phases of the central counter-rotating transmitter wedges relative to their corresponding annular receiver wedge by $\Delta \phi$.

The point-ahead effect due to the linear velocity of the spacecraft, $v_{g}$, can also be compensated for by introducing an additional small angular displacement, $\Delta \psi=2 v_{g} / c$, of the transmitter beam in the forward direction of the spacecraft motion using a prism or mirror at the input to the scanner as in Figure 2. The effectiveness of these point ahead corrections is illustrated in Figure 3 where the circles represent the transmitted laser beam on the ground and the squares represent the projected images of the detector array. Small variations in the spacecraft altitude above the surface can be caused by slight orbital ellipticity, planetary topography, or gravity variations. In our current Mars example, these will cause a maximum decentering of the transmit and receive FOV's near the ground track on the order of $1 \mathrm{~m}$ per \% variation in altitude and much smaller variations near the edge of the scan where the ground beam velocity is significantly reduced.

\section{THE CASE FOR PHOTON COUNTING}

The GLAS instrument weighs about $300 \mathrm{~kg}$ (including two redundant lasers) and is expected to consume approximately $300 \mathrm{~W}$ of spacecraft prime power on average. The GLAS laser is the most powerful of the altimetric lasers currently destined for space and produces an average output power of 4 watts $(100 \mathrm{~mJ} @ 40 \mathrm{~Hz})$ at $1064 \mathrm{~nm}$ with a wallplug electrical efficiency on the order of $5 \%$. Thus, the transmitter is expected to consume about 80 watts. Frequency doubling of the laser radiation would be expected to reduce overall laser power efficiency to less than $3 \%$. It is therefore reasonable to assume that spacecraft prime power resources will limit the average laser output power to a few watts, at least in the near term. Therefore, for the nominal $\mathrm{kHz}$ laser needed for global mapping, the single pulse energy will be restricted to a few $\mathrm{mJ}$ per laser fire.

The mean number of signal photoelectrons detected per laser fire by the receiver from a planar Lambertian diffuse reflector which fills the receiver field of view (FOV) is given by the familiar altimeter equation [Stitch, 1972, Degnan, 2002].

$$
n_{s}=\left[\rho \cos \sigma T_{0}^{2}\right] \frac{\eta_{q} \eta_{r} E_{t} A_{r}}{\pi h v R^{2}}
$$

where the various parameters are defined in Table 1 . Using this expression and the numerical values listed in Table 1 , one can calculate that a $1 \mathrm{~W}$ green laser transmitting 1 $\mathrm{mJ}$ of energy at a 1 nominal $\mathrm{kHz}$ repetition rate will produce approximately 100 photoelectrons per laser fire in a receiver with a $1 \mathrm{~m}$ receive aperture.

If these 100 photoelectrons were somehow distributed equally among the 100 detector pixels by proper shaping and matching of the transmitted laser beam with the projected array on the ground, Poisson statistics predict that over $60 \%$ would be detected if the detection threshold for each timing channel was set at 1 pe (i.e. photon counting mode). 
To achieve greater than $98 \%$ detection probability per channel, one would require about 4 signal pe per pixel in photon counting mode which would translate to $4 \mathrm{~mJ}$ of transmitted energy (or $4 \mathrm{~W}$ of transmitted power at a nominal $1 \mathrm{kHz}$ laser fire rate) assuming the parameters in Table 1 . Smaller receive apertures than the $1 \mathrm{~m}$ assumed here can only be achieved at the expense of more laser energy, and conversely larger imaging telescopes in space would be required to reduce the laser power requirements. Neither is an attractive option, and one must therefore conclude that photon counting is the best near term approach to achieving our measurement goals.

\begin{tabular}{|l|l|}
\hline Parameter & Value \\
\hline Surface Reflectivity, $\rho$ & 0.12 (Soil at $532 \mathrm{~nm})$ \\
\hline Maximum Surface Slope, $\sigma$ & $23^{\circ}$ \\
\hline One-way Atmospheric Transmission, $T_{0}$ & 0.9 \\
\hline Detector Quantum Efficiency, $\eta_{q}$ & $0.4(\mathrm{GaAsP})$ \\
\hline Receiver Optical Throughput, $\eta_{r}$ & 0.4 \\
\hline Photon Energy, $h v$ & $3.74 \times 10^{-19} \mathrm{~J}$ \\
\hline Range to Surface, $R$ & $300 \mathrm{~km}$ \\
\hline Transmitted Energy, $E_{T}$ & $1 \mathrm{~mJ}($ at $532 \mathrm{~nm})$ \\
\hline Receiver Area, $A_{r}$ & $0.785 \mathrm{~m}^{2}(1 \mathrm{~m}$ diameter $)$ \\
\hline Mean Signal Count per Laser fire, $n_{s}$ & $100 \mathrm{pe}$ \\
\hline Exoatmospheric Spectral Irradiance, $N_{\lambda}{ }^{o}$ & $0.087 \mathrm{~W} / \mathrm{m}^{2}-\mathrm{A}^{\circ}(532 \mathrm{~nm}$ at Mars) \\
\hline Receiver FWHM Spectral Bandwidth, $\Delta \lambda$ & $2.5 \mathrm{~A}^{\circ}(\mathrm{GLAS}$ filter) \\
\hline Ground Resolution, $\Delta$ & $5 \mathrm{~m}$ \\
\hline
\end{tabular}

\section{Table 1: Parameters used in Altimeter Link Calculation}

The author has previously provided the theoretical background for a photon counting approach to laser altimetry in which a single photon time-of-flight is used to generate each individual range measurement [Degnan, 2002]. The technique was recently demonstrated on an aircraft using a tiny $\left(8 \mathrm{~mm}^{3}\right)$, low energy $(2 \mu \mathrm{J})$, passively $Q-$ switched microchip $\mathrm{Nd}$ :YAG laser transmitter operating at multi-kHz rates at altitudes up to $6.7 \mathrm{~km}$ [Degnan et al, 2001]. As shown from the raw engineering flight data in Figure 4, the system successfully recorded returns from buildings, trees (plus underlying terrain), and water surfaces and was even able to perform shallow water bathymetry at depths up to two meters. The data in the figure was taken at mid-day in $2 \mathrm{D}$ profiling mode with a quadrant PMT detector, but the system also includes a simple optical wedge scanner, which superimposes a circular scan onto the linear forward motion of the aircraft for generating wide swaths and 3D topographic maps. The mean signal strength per laser fire was on the order of 1 pe or less at $532 \mathrm{~nm}$, and the receiver was designed to provide up to 16 stops per laser fire or 4 per quadrant. The signal was easily extracted from the solar background using post-detection Poisson filtering techniques [Degnan, 2002].

Let us now return to our Mars example. During local daylight operations, a number of false alarms would be recorded due to solar photons scattered from the surface or intervening atmosphere, but these, on average, would also be shared equally among the 
100 receiver channels. The maximum number of solar noise counts per timing channel observed within a range gate, $\tau_{g}$, is given by the expression [Degnan, 2002]

$$
n_{g} \leq\left[N_{\lambda}^{0}(\Delta \lambda)\left(\frac{\Delta}{R}\right)^{2} \tau_{g}\right]\left[\frac{\eta_{q} \eta_{r} A_{r}}{\pi h v}\right]\left[\rho T_{0}^{2}+\frac{1-T_{0}^{2}}{8}\right]
$$

where the first and second terms result from solar scattering off the surface and intervening atmosphere respectively. Using the values in Table 1, we compute slightly less than one noise photoelectron generated for each microsecond of range gate. Thus, the number of timing stops required per channel will be roughly equal to the gate width in microseconds plus one. Of course, the required number of stops can be reduced with a further reduction in the receiver spectral bandwidth. In fact, a photon-counting green receiver on GLAS, designed to view atmospheric scattering from space in parallel with altimetric observations in the infrared, uses a $2.5 \mathrm{~A}^{\circ}$ filter followed by a narrow bandpass etalon which reduces the spectral bandwidth by almost another order of magnitude. It does introduce additional receiver throughput loss, however, which would have a negative impact on the altimetric link discussed here.

An analysis of Eq. (1) indicates that, in the absence of any surface features, the pulse TOF varies by less than 4 nsec peak-to-peak over the full width of the scan. Similarly, since the spacecraft moves only about $3 \mathrm{~m}$ in the roughly $1 \mathrm{msec}$ between successive laser fires, orbital ellipticity and gravity variations will also have a negligible effect on the TOF on this time scale. Thus, the centering of the range gate can be well estimated from the mean TOF observed in the prior laser fire. The width of the range gate, however, must be chosen to accommodate the maximum expected topographic height differential on a spatial scale of $\sqrt{2} N \Delta=70 \mathrm{~m}$, corresponding to the maximum distance between the centers of successive ground spots produced by our scanner. Since each microsecond of range gate corresponds to $150 \mathrm{~m}$ of range uncertainty, a two microsecond range gate should be more than adequate to capture the surface detail on highly sloped $\left(<60^{\circ}\right)$ or highly modulated surfaces. The occasional precipitous slope or cliff will result in a mix of low elevation and high elevation returns, which can be used to initiate a longer range gate for rapid reacquisition at the new elevation.

\section{CONCLUDING REMARKS}

We have argued the feasibility of mapping an entire planet from space with few meter horizontal and decimeter vertical resolution using existing technology or reasonable extrapolations thereof. Our conceptual design incorporates modest power lasers (few mJ at $\mathrm{kHz}$ rate), highly pixellated ( $10 \times 10)$ array detectors with independent photoncounting timing channels, and a novel dual wedge optical scanner which provides for transmitter point-ahead.

Longevity of the laser is clearly still an issue. However, one advantage of low energy, high repetition rate, laser transmitters is that they can be pumped by diode laser arrays 
Longevity of the laser is clearly still an issue. However, one advantage of low energy, high repetition rate, laser transmitters is that they can be pumped by diode laser arrays operating in $\mathrm{CW}$ mode and passively Q-switched by a saturable absorber. Claims of CW laser diode lifetimes in excess of 50,000 hours ( $>5$ years) are not uncommon and, with adequate derating and redundant transmitters onboard, may actually meet the three year mission (26,000 hrs) life assumed here. High energy lasers, on the other hand, are usually pumped by pulse-pumped diodes. Under this mode of operation, the higher peak currents place more electrical and mechanical stress on the diodes, and one is usually delighted to achieve a lifetime of a few billion shots, a number far short of the almost 100 billion needed for a 3 year Mars mission.

If one uses meter aperture receive optics, it is not practical to install the dual wedge scanner in front of the receiver as in Figure 2. Instead, a smaller scanner would be inserted in a common transmit/receive path prior to the final magnifying telescope, e.g. after an annular mirror which reflects the receive energy and passes the transmitter beam through the central hole. To prevent the laser from being blocked by a central obscuration, such as the secondary mirror on a Cassegrain telescope, a small diameter pathway on the order of a centimeter could be provided through the secondary. Alternatively, a obscuration-free design such as an off-axis parabola could be used [Degnan, 2001]. In either case, the wedge angles would be adjusted to compensate for the final magnification in the transmitter and receiver paths so that they create equal conical scans at the output.

Scanner longevity is certainly helped by the use of uniformly rotating components. Momentum wheels have been used for years in space to control the pointing of spacecraft and various instruments. Recent devices with several inch diameters have operated at speeds up to 3000 RPM and have demonstrated lifetimes in excess of four years (http:/www rti org/technology/momen wheel cfm).

\section{REFERENCES}

Abbott, R. and P. Shelus, "Laser observations of the Moon: Identification and construction of normal points for 1969-1971, The Astronomical Journal 78, pp. 784-793, 1973

Abshire, J., E. Ketchum, R. Afzal, P. Millar, X. Sun, "The Geoscience Laser Altimeter System (GLAS) for the ICEsat Mission", Proc. CLEO/QELS 2000, San Francisco, CA, May 7-12, 2000

Beaghler, G., Fibertek, Inc., Herndon, VA, private communication, 2001.

Dubayah, R., J.B. Blair, J.L. Bufton, D. B. Clark, J. JaJa, R. Knox, S. B. Luthcke, S. Prince, J. Weishampel, "The Vegetation Canopy Lidar Mission", Proceedings: Land Satellite Information in the Next Decade II: Sources and Applications, 1997, American Society for Photogrammetry and Remote Sensing, Bethesda, MD, December 1997. 
Degnan, J., "Millimeter Accuracy Satellite Laser Ranging: A Review", in Contributions of Space Geodesy to Geodynamics: Technology, D. E. Smith and D. L. Turcotte (Eds.), AGU Geodynamics Series, Volume 25, pp. 133-162, 1993.

Degnan, J., J. McGarry, T. Zagwodzki, P. Dabney, J. Geiger, R. Chabot, C. Steggerda, J. Marzouk, and A. Chu, "Design and performance of an airborne multikilohertz, photoncounting microlaser altimeter", Int. Archives of Photogrammetry and Remote Sensing, Vol. XXXIV-3/W4, Annapolis, MD, 22-14 Oct. 2001

Degnan, J. "Photon-counting multikilohertz microlaser altimeters for airborne and spaceborne topographic measurements", to be published in J. Geodynamics, 2002.

Ramos-Izquierdo, L., J. Bufton, P. Hayes, "Optical system design and integration of the Mars Observer Laser Altimeter", Applied Optics, vol. 33, pp. 307-322, 1994.

Smith, D., M. Zuber, G. Neumann, and F. Lemoine, "Topography of the Moon from the Clementine Lidar", J. Geophysical Research, Vol. 102, pp. 1591-1611, 1997.

Stitch, M. , "Laser Rangefinding" in Laser Handbook, Eds. F. T. Arecchi and E. O. schulz-Dubois, North Holland Publishing Company, Amsterdam, pp. 1745-1804, 1972.

Vasile, S., P. Gothoskar, R. Farrell, D. Sdrulla, "Photon detection with high gain avalanche photodiode arrays", IEEE Trans. Nuclear Science, vol. 45, pp. 720-723, 1997. 


\section{FIGURE CAPTIONS}

1. Conceptual design of a planetary mapper showing the projection of the square detector array onto the surface circumscribed by the circular laser spot. A dual optical wedge scanner allows the instrument to fill in the gaps between ground tracks. Pointahead compensation within the scanner allows the detector array to be centered on the area illuminated by the laser as the photons arrive one roundtrip transit time after the laser fires.

2. A dual wedge optical scanner with point-ahead compensation.

3. Ground pattern created by the dual wedge scanner with a starting phase offset of 90 degrees between the two counter-rotating plates. The squares represent the detector array and the circles represent the laser transmit beam on the ground. Their centers coincide because of compensation for the spacecraft forward velocity and the rotational phase lag by the round trip transit time of the photons.

4. Engineering flight configuration plus sample profiling data from: small buildings and trees in the town of Chincoteague, VA, from $6.7 \mathrm{~km}$ altitude; tree lines and underlying terrain from $3.4 \mathrm{~km}$ altitude; and shallow water bathymetry over the Chesapeake Bay from $3.4 \mathrm{~km}$ altitude. 


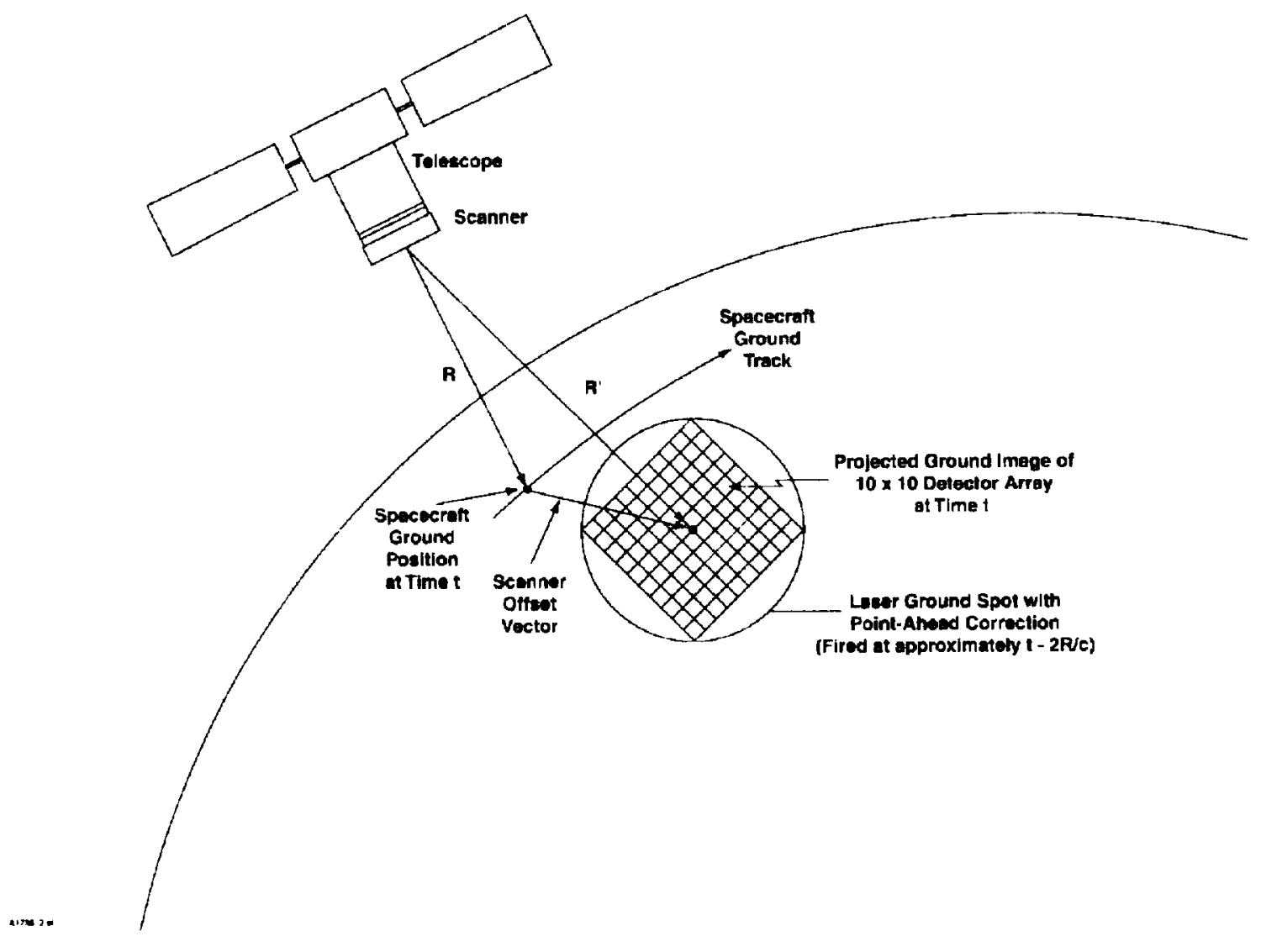




\section{Dual Wedge Optical Scanner with Transmitter Point-Ahead \\ Correction}
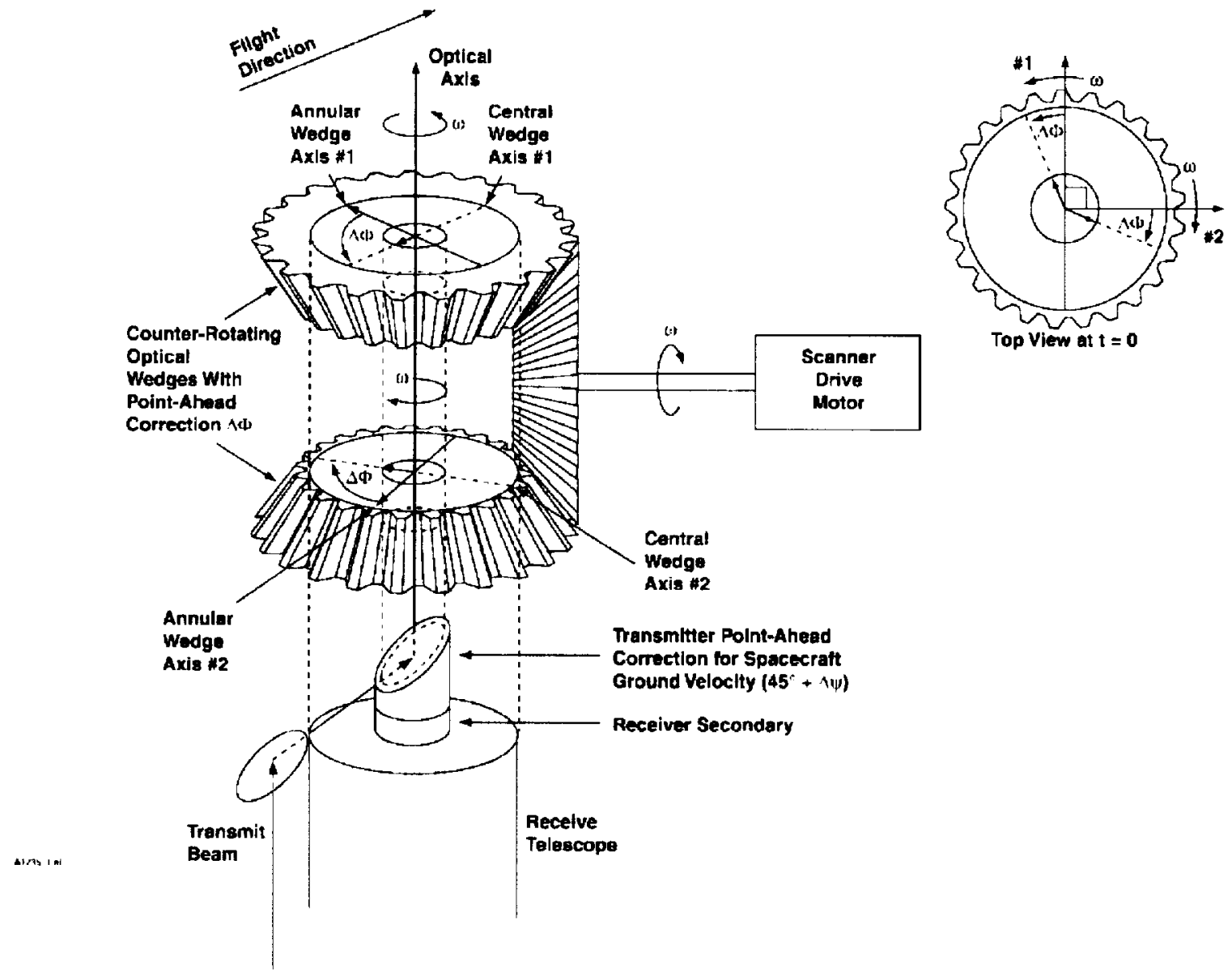


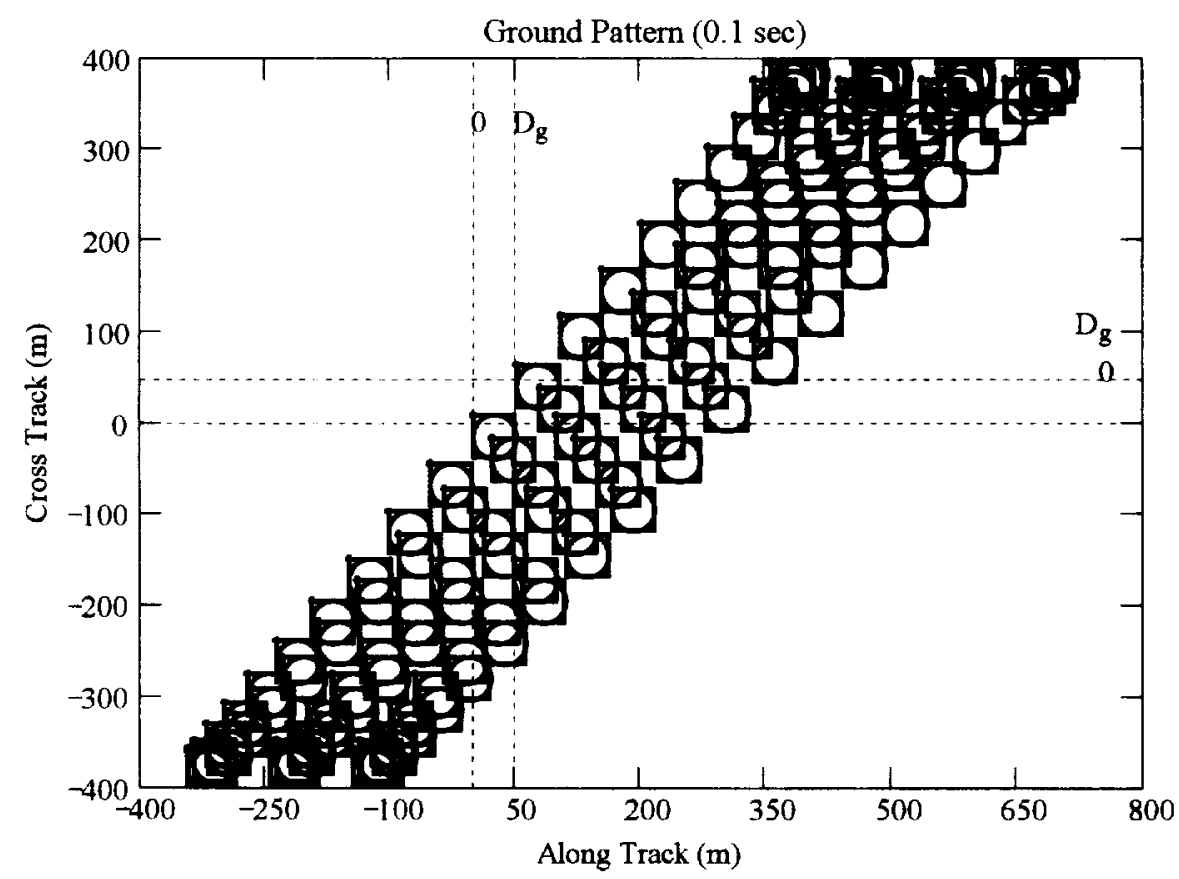

$\mathrm{D}_{\mathrm{g}}=50 \mathrm{~m}$ 
- Engineering Flight Parameters

NASA P-3 Aircraft. Wallops Flight Center

Locale: Chincoteague, VA \& Chesapeake Bay

Flight Altitudes: 3.5 to $6.7 \mathrm{~km}(11,000$ to $22,000 \mathrm{ft})$

Early afternoon (maxim um solar background)

Laser Energy: $<2 \mu \mathrm{J}$ (a) $532 \mathrm{~nm}$

- Laser Repetition Rate: $\mathbf{3 . 8 \mathrm { kHz }}$

Laser Power: $\sim 7 \mathrm{~mW}$

Effective Telescope Diameter: $14 \mathrm{~cm}$

Mean Signal Strength per Laser Fire: $\sim 0.88$ pe

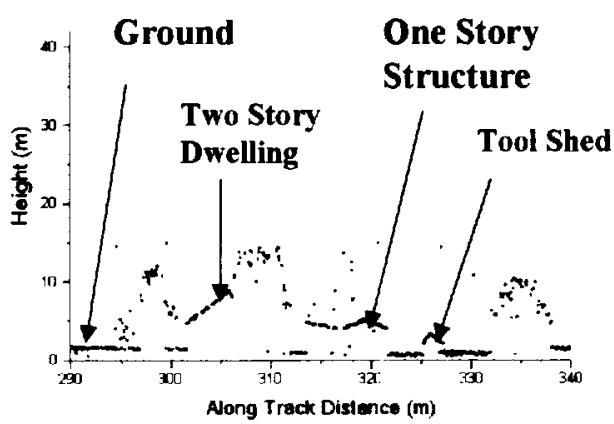

\section{Buildings and Trees}

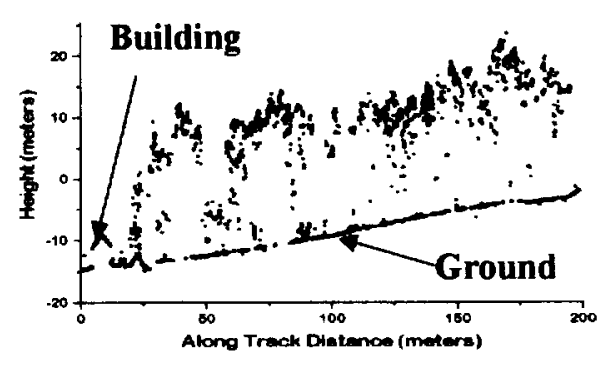

Tree Canopy Heights

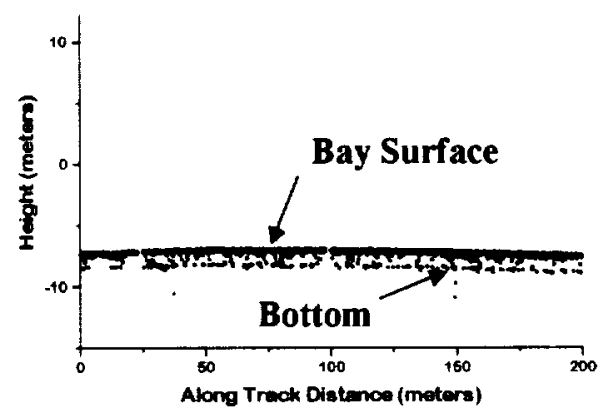

Shallow Water Bathymetry 